\title{
Five-parameter grain boundary distribution in grain boundary engineered brass
}

\author{
Chang-Soo Kim ${ }^{\text {a }}$, Yan $\mathrm{Hu}{ }^{\mathrm{b}}$, Gregory S. Rohrer ${ }^{\mathrm{a}}$, Valerie Randle ${ }^{\mathrm{b}, *}$ \\ a Department of Materials Science and Engineering, Carnegie Mellon University, Pittsburgh, PA 15213-3890, USA \\ ${ }^{\mathrm{b}}$ Materials Research Centre, School of Engineering, University of Wales Swansea, Singleton Park, Swansea SA2 8PP, UK
}

Received 10 November 2004; accepted 18 November 2004

Available online 15 December 2004

\begin{abstract}
We report the first application of a five-parameter determination of grain boundary types to grain boundary engineered alphabrass. The most important findings were that the distribution of planes showed a prevalence of $\langle 110\rangle$ tilt boundaries, especially asymmetric tilt types, and the presence of $\langle 1111\rangle$ twist boundaries. This distribution resulted from the low energy of these boundary types.
\end{abstract}

(C) 2004 Acta Materialia Inc. Published by Elsevier Ltd. All rights reserved.

Keywords: Grain boundary energy; Twinning; Misorientation; Grain boundary plane; Electron backscatter diffraction (EBSD)

\section{Introduction}

It is now known that if the types of grain boundaries in polycrystalline materials can be controlled, certain material properties can be improved. In particular, processing designed to give prolific multiple annealing twinning in low stacking-fault energy face-centred cubic metals and alloys has been successfully used for property enhancement. This practice has come to be known as 'grain boundary engineering' and has been known to mitigate intergranular stress corrosion cracking in nickel-based alloys [1] and to influence mechanical behaviour such as ductility [2]. Grain boundary engineering has recently been reviewed in detail [3].

Many reports of grain boundary engineered (GBE) materials cite an enhanced proportion of low- $\Sigma$ coincidence site lattice (CSL) boundaries, where $\Sigma$ is the reciprocal density of coinciding sites, as the factor responsible for property improvements. However, prac-

\footnotetext{
* Corresponding author. Tel.: +44 1792 295841; fax: +44 1792 295676.

E-mail address: v.randle@swansea.ac.uk (V. Randle).
}

tically every CSL in GBE materials is $\Sigma 3$ or $\Sigma 3^{n}$, where annealing twins are a subset of the $\Sigma 3$ CSL class. $\Sigma 3$ s are the only CSL class known, in bulk three-dimensional polycrystals, consistently to exhibit 'special' (i.e., better than average) properties. It is, therefore, reasonable to assume that twinning is instrumental in the property improvements. The twins themselves do not constitute part of the grain boundary network; rather they modify the network where they join it. How this process sometimes produces dramatic improvements in properties is not yet completely understood.

Further progress in understanding the mechanisms of GBE requires a more in-depth knowledge of grain boundary structure. GBE materials are usually characterized on the basis of the three lattice misorientation parameters. However, to identify the boundaries of high planar coincidence that are thought to be special, it is also necessary to determine the two parameters that specify the grain boundary plane. Recently a method has been designed to measure all five grain boundary parameters. In the first instance, the method required serial sectioning to obtain the inclination of boundary surfaces [4]. More recently, a stereological method has been 
devised to extract the relative populations of grain boundaries as a function of all five grain boundary parameters from orientation maps in a single planar section [5]. In this paper, we report the first application of the five-parameter determination of grain boundary types to a grain boundary engineered material, namely alpha-brass.

\section{Experimental}

Specimens of alpha-brass underwent GBE processing by five iterations of $25 \%$ uniaxial strain followed by annealing in air for $300 \mathrm{~s}$ at $665^{\circ} \mathrm{C}$. Full details of the processing, specimen preparation and effect on properties have been published elsewhere [6]. Many high resolution electron back-scatter diffraction (EBSD) orientation maps of the specimen were obtained using HKL Channel 5 software interfaced to a Philips XL30 scanning electron microscope operated at an accelerating voltage of $20 \mathrm{kV}$. Maps were obtained by beam scanning followed by stitching together contiguous regions. A step size of $2 \mu \mathrm{m}$ was used for mapping. Approximately 20,000 grains comprised the total dataset.

The orientation data were used to determine the grain boundary character distribution, $\lambda(\Delta g, n)$, which is defined as the relative areas of distinguishable grain boundaries characterized by their lattice misorientation $(\Delta g)$ and boundary plane orientation (n) [7]. The grain boundary character distribution is measured in multiples of a random distribution (MRD). Each grain boundary connecting two triple points can be broken up into to several line segments, referred to as grain boundary traces. The direction of the trace and the lattice misorientation across each trace specify four of the five parameters necessary to determine the distribution: the only unknown parameter is the inclination of the boundary plane with respect to the surface. If a sufficient number of grain boundary traces from randomly orientated, symmetrically indistinguishable bicrystals are observed, then stereology can be used to specify the probability that certain grain boundary planes appear in the microstructure [5]. In this case, our data set was made up of 77,000 grain boundary traces extracted from the orientation maps using a procedure described by Wright and Larsen [8]. The grain boundary character distribution, $\lambda(\Delta g, n)$, is parameterized and discretized as described in our previous work, and therefore, has a resolution of approximately $10^{\circ}[4]$.

\section{Results}

The grain boundary misorientation statistics derived from the orientation maps are given in Table 1. The HKL and TSL software yielded consistent misorienta-
Table 1

Proportions of $\Sigma$ boundaries and low angle boundaries in grain boundary engineered brass specimens

\begin{tabular}{lcc}
\hline & \% By length & \% By number \\
\hline$\Sigma 3$ & 57.7 & 38.2 \\
$\Sigma 9$ & 3.4 & 6.7 \\
$\Sigma 27 \mathrm{a}$ and $\Sigma 27 \mathrm{~b}$ & 1.9 & 3.9 \\
Low angle & 2.6 & 2.9 \\
\hline
\end{tabular}

tion statistics. More than half of the total boundary length in the map was $\Sigma 3$. Such prolific twinning resulted in the generation of higher order twins, particularly $\Sigma 9$ and $\Sigma 27$, but these boundaries tended not to survive in the microstructure in large numbers because interaction events with other $\Sigma 3^{n} \mathrm{~s}$ regenerated $\Sigma 3 \mathrm{~s}$, and hence there is only $3.4 \% \Sigma 9$ and $1.9 \% \Sigma 27$ (roughly equally partitioned between $\Sigma 27$ a and $\Sigma 27$ b) by length [9]. It is evident from the data in Table 1 that the $\Sigma 3$ boundaries make up a greater fraction of the total boundary length than they do the total number of boundaries. This demonstrates that they are, on average, longer than the other boundaries. The dominance of multiple twinning in the microstructure of the GBE brass can be clearly seen in Fig. 1, which is one of the orientation maps, where general (random) boundaries are represented in black and $\Sigma 3^{n}$ boundaries and low angle boundaries $(1 \leqslant n \leqslant 3)$ are grey. There were no other CSLs present at levels above that expected for random generation. The crystal orientations are randomly distributed, which is a consequence of multiple twinning.

Following the five-parameter analysis, the boundary plane populations, $\lambda(\Delta g, n)$, are plotted in stereographic projection as multiples of a random distribution (MRD). Fig. 2a shows the distribution of boundary plane normals, $\lambda(n)$, independent of the lattice misorientation for the entire data set, which is made up of more than 77,000 line segments. The population is very high

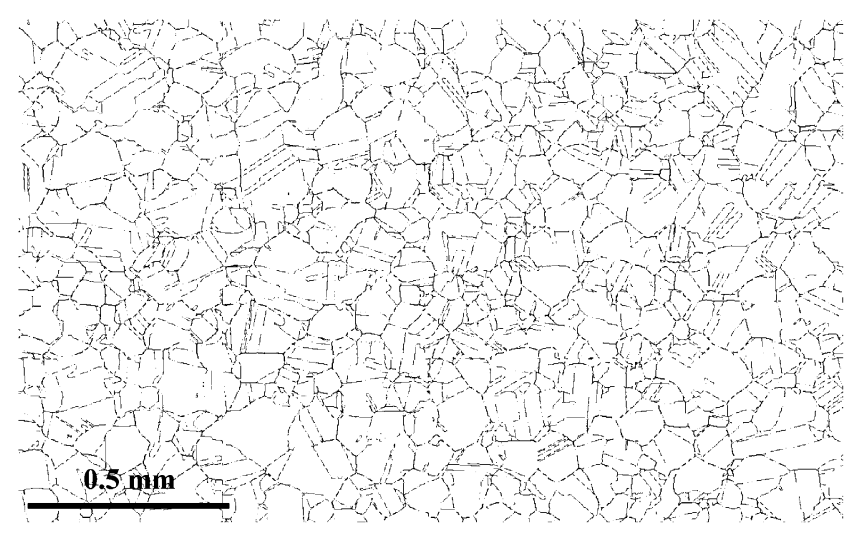

Fig. 1. One of the orientation maps from grain boundary engineered brass. General (random) boundaries are represented in black and $\Sigma 3^{n}$ boundaries and low angle boundaries $(1 \leqslant n \leqslant 3)$ are grey. 


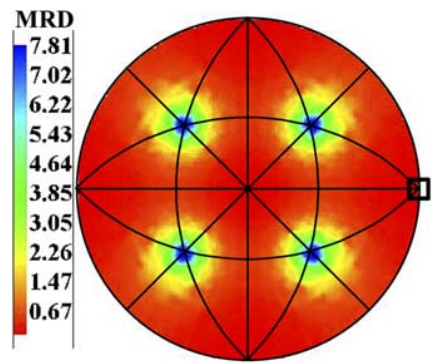

(a)

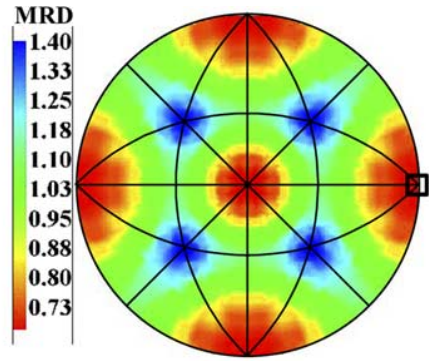

(b)

Fig. 2. A plot of $\lambda(n)$, the distribution of grain boundary planes in grain boundary engineered brass: (a) all observations; (b) all boundaries more than $10^{\circ}$ from the $\Sigma 3$ misorientation. The data are plotted in stereographic projection along [001]. The [100] direction is in the plane of the page and marked by a square.

at a single peak centred on $\langle 111\rangle$. This result is not surprising given that more than half the interface length is $\Sigma 3$ and the morphology of these boundaries in the map indicates that most of them are coherent twins on $\{111\}$. In fact, the coherent twins dominate the distribution. It is more helpful, therefore, to examine that subset of the population that excludes $\Sigma 3 \mathrm{~s}$, as shown in Fig. $2 \mathrm{~b}$. This subset of the population consists of approximately 47,000 line segments, which are sufficient to produce reliable grain boundary plane statistics [5]. It can be seen that the distribution has retained the same form even after $\Sigma 3$ s are removed, namely a single peak on $\{111\}$ planes, although in the $\Sigma 3$-excluded distribution the population is reduced from 7.8 MRD to $1.4 \mathrm{MRD}$ and this makes it possible to resolve the structure of the population at other orientations. The data in Fig. 2 show that $\{100\}$ boundary planes are observed with a fre- quency that is less than that expected in a random distribution.

It is revealing to view the distribution of boundary planes at specific lattice misorientations with low index misorientation axes. Fig. 3 shows the distribution of planes at six misorientations about the $\langle 110\rangle$ axis. The population statistics have been calculated after excluding the $\Sigma 3$ misorientation, whose large population unduely influences the MRD values throughout the five-parameter space. The position of the [1 10 l misorientation axis is marked on the stereograms. It is quite striking that for all misorientation angles the boundary planes lie preferentially on the zone of [110] (that is, they are tilt boundaries) with the maximum MRD in each plot ranging from nearly 4 to nearly 27 . Although this trend is apparent in all the angle ranges, it is strongest at the $\Sigma 27 \mathrm{a}$ and $\Sigma 9$ misorientations. For

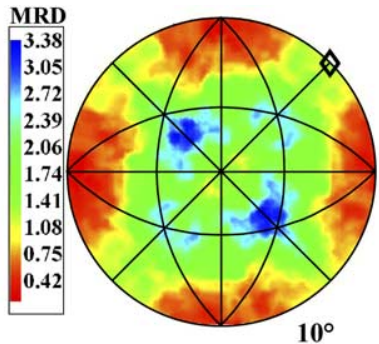

(a)

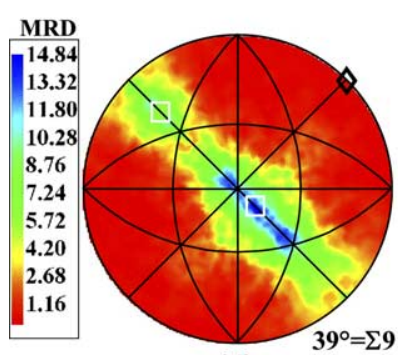

(d)

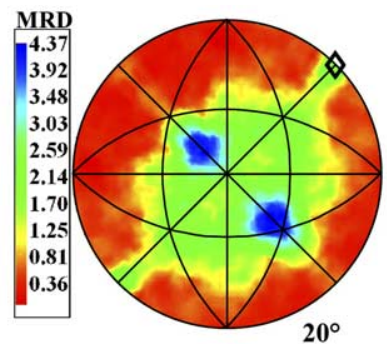

(b)

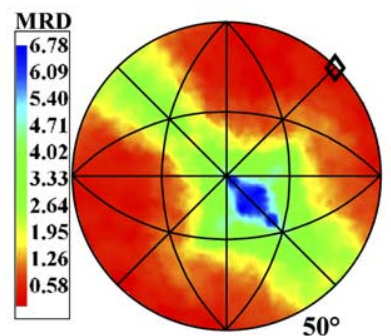

(e)

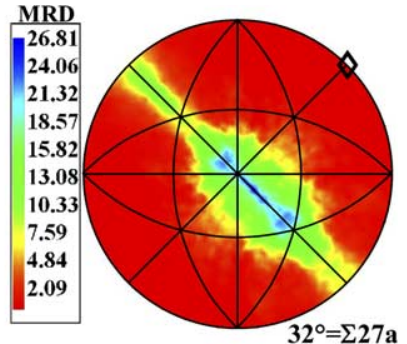

(c)

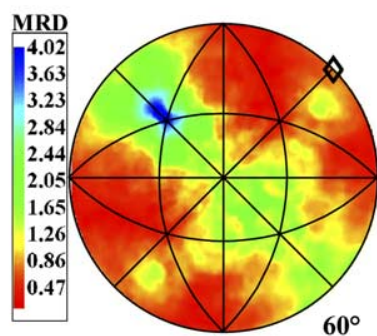

(f)

Fig. 3. Distribution of grain boundary planes for [1 10$]$ misorientations. The [1 10$]$ misorientation axis, in the plane of the page, is marked by a diamond in (a) and the data are plotted in stereographic projection along [00 1$]$. (a) $\lambda\left(n \mid 10^{\circ} /[110]\right)$, (b) $\lambda\left(n \mid 20^{\circ} /[110]\right),\left(\right.$ c) $\lambda\left(n \mid 32^{\circ} /[110]\right),(d) \lambda\left(n \mid 39^{\circ} /\right.$ [1 10$])$, (e) $\lambda\left(n \mid 50^{\circ} /[110]\right)$, and (f) $\lambda\left(n \mid 60^{\circ} /\left[\begin{array}{lll}1 & 10\end{array}\right)\right.$. In (d), the positions of the symmetric tilts are marked with squares. 


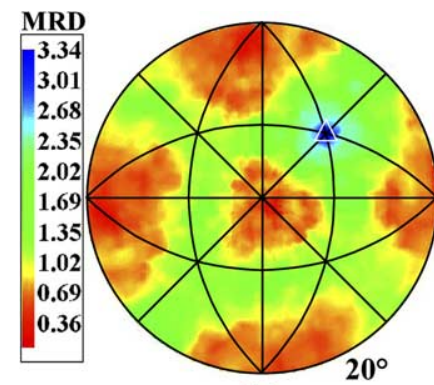

(a)

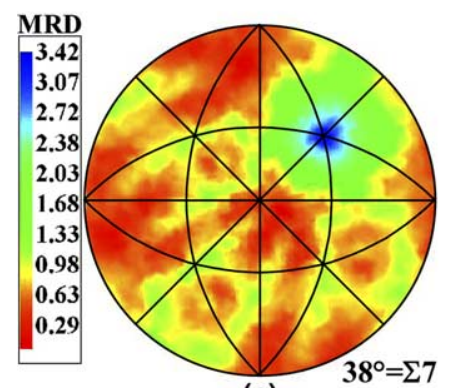

(c)

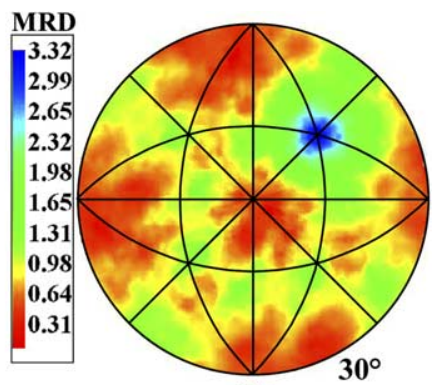

(b)

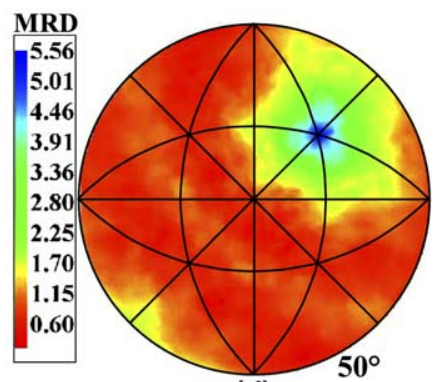

(d)

Fig. 4. Distribution of grain boundary planes for [1 111$]$ misorientations. The position of the [ 1111$]$ misorientation axis is marked by a triangle in (a) and the data are plotted in stereographic projection along [001]. (a) $\lambda\left(n \mid 20^{\circ} /[111]\right)$, (b) $\lambda\left(n \mid 30^{\circ} /[111]\right)$, (c) $\lambda\left(n \mid 38^{\circ} /[111]\right),\left(\right.$ d) $\lambda\left(n \mid 50^{\circ} /[111]\right)$.

misorientations less than $60^{\circ}$, there is more than one maximum on each of the [110] zones, indicating that the tilt boundaries are asymmetric.

Fig. 4 shows the population of boundary planes for selected misorientations about $\langle 111\rangle$. As in Fig. 3, $53 \mathrm{~s}$ have been excluded from the sample population because the peak for (111) planes at $60^{\circ} /\langle 111\rangle$, the coherent twin, is more than $2200 \mathrm{MRD}$, and this affects the resolution in the other angle plots. Fig. 4 shows that for each misorientation angle, there is a single peak at (111) with an MRD greater than 3.3. The populations for boundaries with $\langle 100\rangle$ misorientations (not shown) were all less than 1 MRD.

As a consequence of the multiple twinning $\Sigma 27 \mathrm{~b}$, in addition to $527 \mathrm{a}$, is present in the data at levels higher than expected for random generation. Fig. 5 shows the distribution of planes for $\Sigma 27 \mathrm{~b}$, where the disorientation

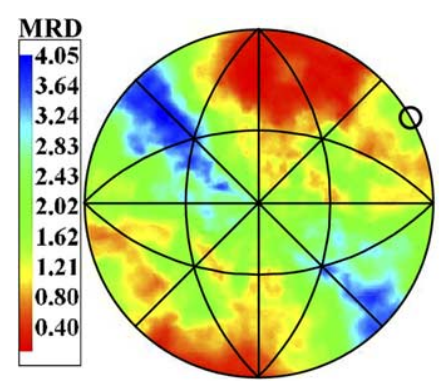

Fig. 5. $\lambda\left(n \mid 35^{\circ} /[210]\right)$, the distribution of grain boundary planes for $\Sigma 27 \mathrm{~b}$ lattice misorientation. The position of the [210] misorientation axis is marked by a circle and the data are plotted in stereographic projection along [0 0101$]$. is $35^{\circ} /\langle 210\rangle$, and it can be seen that there is a zone of planes which corresponds broadly to the $\langle 110\rangle$ zone. The peaks on this zone are up to 4 MRD. The peaks, which correspond to boundaries that are not ideal tilts, are more diffuse than those for misorientations about $\langle 110\rangle$ and $\langle 111\rangle$, as shown on Figs. 3 and 4.

\section{Discussion}

The most striking points to arise from the analysis of grain boundary plane data are the prevalence of $\langle 110\rangle$ tilt boundaries, especially asymmetric tilt types, and the presence of $\langle 111\rangle$ twist boundaries. Previous work has highlighted the importance of asymmetric boundaries, as opposed to symmetric types, in polycrystals $[10,11]$. From a probability point of view, there are many more possible asymmetric boundaries than symmetric ones, even when only tilt boundaries and low index planes are considered. For example, misorientations about $\langle 110\rangle$ give rise to two symmetric boundaries on $\{111\}$ and $\{112\}$ ( $\Sigma 3$ coherent and incoherent twins, respectively) and many asymmetric boundaries such as $\{411\}\{110\},\{511\}\{111\},\{221\}\{100\},\{211\}\{552\}$, $\{221\}\{744\}$, etc., [12]. Geometrical constraints dictate that an asymmetrical boundary usually comprises only one low-index plane, because once the indices of the plane on one side of the boundary are selected, the indices of the second plane are fixed according to the misorientation. Fig. 3 shows evidence that often one plane is near $\{111\}$. There is no evidence in Fig. 3 of a strong preference for the symmetrical planes of $\Sigma 9$ and $\Sigma 27 \mathrm{a}$, 
which are $\{411\}$ and $\{221\}$ in the case of $\Sigma 9$ and $\{721\}$ in the case of $\Sigma 27 \mathrm{a}$. While the populations of the symmetric planes is relatively high, they are not greater than the populations of the asymmetric planes in the same zone.

In the present data, we can extract from the orientation maps that more than $70 \%$ of interfaces are misoriented on $\langle 110\rangle$, and of those the majority are asymmetric tilt or near tilt types. A preference for asymmetrical tilt boundaries has been noted previously both from high resolution electron microscopy observations [10] and from EBSD-based measurements of boundary planes [13]. Based on previous work, which has shown an inverse relationship between the frequency with which boundaries occur in the population and their energy, we assume that boundary types with high populations are also low energy boundaries [4,14-16]. This assumption is supported by the results of calculations which have shown that asymmetric boundaries may have low energies when bounded by low-index planes [16] and that $\langle 110\rangle$ tilts in particular lie in an energy valley [12]. Therefore, in the present data, it is reasonable to conclude that $\langle 110\rangle$ tilt and near tilt boundaries have low energies and that this is the reason for their prevalence.

The presence of high proportions of $\langle 111\rangle$ twist boundaries is probably also associated with a low energy configuration, since the presence of two low index planes such as $\{111\}$ at a grain boundary implies a lower than average energy, even when they are rotated with respect to one another [16]. It should be noted that the boundary population at the $\left\{\begin{array}{lll}1 & 1 & 1\end{array}\right\}$ twist position at the $\Sigma 7$ misorientation is not significantly different from the other orientations that do not correspond to CSL boundaries. In other words, apart from the $\Sigma 3$ misorientation, the population of low energy $\{111\}$ twists is roughly independent of the misorientation angle and the high population of these types of boundaries should not be ascribed to coincidence relations, but to the presence of the low index planes. Similarly, apart from $\Sigma 9$ and $\Sigma 27 \mathrm{a}$, the asymmetric tilts on $\langle 110\rangle$ do not correspond to CSLs. Finally, the distribution of planes for $\Sigma 27 \mathrm{~b}$ boundaries is interesting since it appears to lie on the $\langle 110\rangle$ zone. This might be because a symmetry-related solution for the $\Sigma 27 \mathrm{~b}$ disorientation is $146^{\circ} /\langle 771\rangle$, and this axis is only $5.8^{\circ}$ from $\langle 110\rangle$.

\section{Conclusions}

The distribution of grain boundary misorientations and planes has been acquired for more than 20,000 grains in grain boundary engineered brass. More than half of the interface length was $\Sigma 3$. The most important findings of the investigation are that the distribution of planes showed a prevalence of $\langle 110\rangle$ tilt boundaries, especially asymmetric tilt types, and the presence of $\langle 111\rangle$ twist boundaries. This distribution is a consequence of the low energy of these types of boundary. This is the first time that the distribution of grain boundary planes has been measured in a grain boundary engineered material.

\section{Acknowledgments}

The work at Carnegie Mellon University was supported primarily by the MRSEC program of the National Science Foundation under Award Number DMR-0079996. The work at Swansea was partially supported by the Engineering and Physical Sciences Research Council.

\section{References}

[1] Lin P, Palumbo G, Erb U, Aust KT. Scripta Met Mater 1995;33:1387.

[2] Randle V. Acta Mater 2004;52:4067.

[3] Randle V. Mater Charact 2001;47:411.

[4] Saylor DM, El-Dasher BS, Rollett AD, Rohrer GS. Acta Mater 2004;52:3649.

[5] Saylor DM, Adams BL, El-Dasher BS, Rohrer GS. Metall Mater Trans 2003;34A:1.

[6] Randle V, Davies H. Mater Trans 2002;33A:1853.

[7] Rohrer GS, Saylor DM, El-Dasher BS, Adams BL, Rollett AD, Wynblatt P. Z Metall 2004;95:197.

[8] Wright SI, Larsen RJ. J Microsc 2002;205:245.

[9] Randle V. Acta Mater 1999;47:4187.

[10] Merkle KL. Scripta Metall 1989;23:1487.

[11] Merkle KL. Ultramicroscopy 1991;37:130.

[12] Wolf U, Ernst F, Muschik T, Finnis MF, Fischmeister HF. Philos Mag 1992;66A:991.

[13] Randle V. Mater Sci Technol 1999;15:246

[14] Saylor DM, Morawiec A, Rohrer GS. Acta Mater 2003;51:3675.

[15] Saylor DM, El-Dasher BS, Sano T, Rohrer GS. J Amer Ceram Soc 2004;87:670

[16] Merkle KL, Wolf D. Philos Mag 1992;65A:513. 